\title{
Infected Vertebroplasty Due to Uncommon Bacteria Solved Surgically: A Rare and Threatening Life Complication of a Common Procedure
}

\section{Report of a Case and a Review of the Literature}

Matias Alfonso Olmos, MD, PhD, Alvaro Silva González, MD, Julio Duart Clemente, MD, PhD, and Carlos Villas Tomé, MD, PhD

From the Department of Orthopaedic Surgery, University Clinic of Navarra, Navarra, Spain.

\section{ABSTRACT}

Address correspondence and reprint requests to Matías Alfonso Olmos, $\mathrm{MD}, \mathrm{PhD}$

Pío XII 36, Pamplona, Navarra, Spain, 31080;

E-mail: malfonsool@unav.es

Study Design: Case report.

Objective: The aim of this work is to describe a case of infected vertebroplasty dueto uncommon bacteria solved surgically with 2 years of follow-up and to discuss 6 other cases found in literature.

Summary of Background Data: Vertebroplasty is a well-known and useful technique for the treatment of painful osteoporotic vertebral fractures. Complications, such as cord or root compression or pulmonary embolisms, are infrequent and are mainly related with the frequent escape of cement throughout the vertebral veins. Infection is even more rare, but when it occurs is difficult to manage and can be a life-threatening complication.

Methods: A 63-year-old-man had a spondylitis of L2 after vertebroplasty. The patient was initially managed with antibiotics without clinical improvement. Surgical treatment by anterior debridement and anterior and posterior stabilization was done. The bacteria isolated from the intraoperative cultures were Serratia marcescens, Stenotrophomonas maltophilia, and Burkholderia cepacia. After surgery, the patient was treated with antibiotics for 3 month.

Results: After 2 years of follow-up, the patient was free of pain, without signs of infection, and a correct fusion was achieved.

Conclusion: When facing an infected vertebroplasty, initial conservative treatment with needle biopsy culture and antibiotic administration are a rational option to start. If this treatment fails, surgical debridement is then indicated in order to remove the infected tissue and the acrylic cement and to stabilize the spine. Although this can be an effective treatment, it could be a difficult and hazardous surgical procedure.

Key words: infected vertebroplasty, adult, spondylitis, PMMA, vertebroplasty, complication. 


\section{INTRODUCTION}

Vertebroplasty is a well-known and useful technique for treating some painful osteoporotic compression fractures, vertebral malignancy, and painful hemangiomas. ${ }^{1-6}$

There is a strong evidence of improvement of quality of life after this procedure. Relief of pain after vertebroplasty is more than the $90 \%$ throughout different series. ${ }^{7,8}$

Reported complications associated with this procedure are between $1 \%$ and $3 \%$ for osteoporotic fractures, $2.5 \%$ for hemangiomas, and up to $10 \%$ for metastatic vertebral lesions. ${ }^{2,9,10}$ Although the majority of these problems are minor complications, some serious events, for example, permanent neurologic deficit, pulmonary embolism, serious infections, or even death, may occur.

In this report, we present a case of a postvertebroplasty spondylitis, which did not improve with conservative treatment and was treated successfully by surgical debridement, spinal stabilization, and antibiotic administration. We discuss its significance and we compare it with 6 other cases found in the literature.

\section{CASE REPORTS}

We present the case of a 63-year-old man, without significant medical history, who had a burst fracture of the inferior endplate of L3 from a car accident. The patient was first treated in another hospital with orthosis for 3 months without clinical improvement; later on, a vertebroplasty with polymethylmethacrylate (PMMA) was performed inside an angiographic room. During the procedure, prophylaxis with cephalosporin was administrated. After vertebroplasty, the patient improved only for 10 days, until he started with fever and back pain. Magnetic resonance imaging (MRI) study was compatible with infection, but a needle biopsy-culture was negative. Although the patient received antibiotic treatment (ceftazidime and ciprofloxacin), no improvement was observed, being transferred 1 month later to our center for further care with the diagnosis of spondylitis of L3. At that moment, the patient complained of back pain, fever, and weight loss. Blood cell count (WBC) was $7100 / \mu \mathrm{L}$, with $39.3 \%$ segmented neutrophils, erythrocyte sedimentation rate (ESR) was $70 \mathrm{~mm} / \mathrm{hr}$ and C-reactive protein (CRP) was $2.5 \mathrm{~g} / \mathrm{dL}$. Lumbar radiographs showed partial filled of L3 (Figure 1); MRI showed infection signs around L3 with edema in adjacent vertebrae (Figure 2). Blood cultures and CT-guided needle biopsyculture were negative. Empiric antibiotic treatment with teicoplanin and levofloxacin was indicated without observing improvements of clinical or laboratory parameters during the first week. Surgical debridement was then indicated: First, we performed an anterior retroperitoneal approach, and a piecemeal corpectomy of L3 was done, in order to remove infected PMMA. Later on, an iliac crest autograft was placed (Figure 3). During this surgery, we had to deal with a cava vein thrombosis and a left arterial iliac occlusion due to extended retractor compression, both complications solved successfully by a vascular surgeon. 
One week later, we performed a posterior approach, L1-L5 posterolateral fusion with pedicular screws on L1 and L5, sublaminar wiring at L2 and L4, rod fixation, and autograft. We preferred to use sublaminar wiring on adjacent L3 vertebrae to avoid contact to those vertebral bodies that showed edema on the MRI of possible pyogenic origin.

Seven days later, we observed a mobilization of the iliac graft; we had to replace the graft and fix it with a screw. Intraoperative bone cultures were positive for Serratia marcescens, Stenotrophomonas maltophilia, and Burkholderia cepacia. The patient was finally treated with oral antibiotics (levofloxacin ant cotrimoxazol) and orthosis for 3 months. Two years later, the patient was free of pain, without signs of infection, and a correct vertebral fusion was observed (Figure 4).

\section{DISCUSSION}

Since Galibert and Deramond ${ }^{11}$ introduced this procedure, a small complication rate has been published, most without medical consequences.

Despite cement leakage to disc space or through the venous drainage system is very frequent, symptomatic compression of roots in the neural foramens, or cord compression are less frequent and usually are treatable successfully with steroids. Postvertebroplasty emergency decompression surgery is exceptional. Weill et al reported 1 case with persistent sciatica due to cement epidural leak that was solved with surgical decompression. ${ }^{9}$ Cotton et $\mathrm{al}^{2}$ reported 3 patients that required surgery for postvertebroplasty compression radiculopathies.

Other complications, such as pneumothorax, fatty embolism, psoas hematoma, dural sac leakage, pedicle fracture, ribs fractures, intradiscal or subcutaneous placement of cement, anaphylactic reaction, and death, are also exceptional. ${ }^{7,12-14}$ Transient postoperative in-crease of pain after vertebroplasty had been observed in some cases of metastases. $^{15}$

Migration of cement throughout the cava vein like emboli is at this time the most frightening complication; nevertheless, its occurrence is anecdotal. Padovani et $\mathrm{al}^{16}$ reported 1 patient with a confirmed pulmonary embolism due to cement, treated successfully with anticoagulant therapy. Jensen et $\mathrm{al}^{7}$ reported 2 presumed pulmonary embolisms without clinical manifestation.

Infection after vertebroplasty is considered very rare. Until this review, we found only 6 cases published:

Deramond et al reported a case of spondylodiscitis after vertebroplasty of a metastases in an immunosuppressed patient; the patient was treated with long-term antibiotics, not needing surgery. ${ }^{15}$

Kallmes and Jensen ${ }^{17}$ documented one infection in 63 vertebrae of 41 patients treated with vertebroplasty. The patient was immunocompromised from high-dose steroids. The authors used tobramycin added to the cement and intravenously administered 
antibiotics; Staphylococcus epidermidis was isolated. There are no data about the follow-up of this patient.

Yu et $\mathrm{al}^{18}$ reported one vertebral osteomyelitis after vertebroplasty of a T12 osteoporotic fracture. The patient had a history of urinary tract infection treated with intravenous cefazolin and gentamycin drugs for 1 week before vertebroplasty. One month after the procedure, the patient became febrile and vertebral infection was confirmed by MRI and blood test. The patient was operated on surgically by anterior and posterior approach with corpectomy, interbody strut graft. and posterolateral fusion with pedicular screws. No bacteria were isolated; antibiotics were administered for 6 months. Good outcome was observed at 15 months of follow-up.

Walker et $\mathrm{al}^{19}$ reported 2 patients that had osteomyelitis after vertebroplasty. One patient, a 64-year-old woman, had a history of urinary tract infection and secondary sepsis, treated with intravenous antibiotics for 3 weeks before the vertebroplasty. The patient became febrile 11 days after the surgery; bone and blood cultures were positive to Enterobacter species.

The other patient, a 49-year-old woman, had a previous history of discectomy on T12L1 performed to treat a discitis and 1 year later had an interbody fusion with cage over the same level T12-L1. One year from the last surgery, a vertebroplasty was performed for the treatment of an osteoporotic fracture of L3. Eight months after the procedure, infection was found; tissue cultures revealed Staphylococcus aureus within the L3 body and on the surface of the PMMA.

Both patients were treated by anterior approach, corpectomy interbody titanium cage filled with autograft, later posterior approach with pedicular screws stabilization, and long-term antibiotic treatment. At least 1 patient did well at 9 months of follow-up.

Schmid et $\mathrm{al}^{20}$ reported a 55-year-old man with previous medical history of liver cirrhosis who developed spondylitis after three lumbar vertebroplasties. A paravertebral abscess was aspirated without bacteria identification. The patient was managed with intravenous ciprofloxacin and consecutive clindamycin for 3 month. No surgery was needed despite further collapse of L5. Infection signs disappeared after 1 year of followup; nevertheless, the patient had slight chronic lumbar pain.

Our patient had no history of previous infection, surgery, or immunosuppression. The type of bacteria found, considered as nonpathogenic saprophyte, leads us to believe that the source of the infection was contamination during the procedure. This type of bacteria is also considered a nosocomial highly multiresistant to antibiotics agents, although having a small virulence they are able to produce serious and difficult to manage osseous infections, especially in immunosuppressed patients. $^{21}$

Considering that the bacteria would have been seeded during the procedure, it is remarkable that they would have resisted the elevated temperature that reached the bone cement during polymerization.

We are not able to establish whether the cause of contamination was lack of asepsis during the procedure or just because of performing such vertebroplasty inside a radiologic room, probably not as clean as an operation room. 
We always perform vertebroplasty inside an operation room, using local anesthesia in one or two levels or total anesthesia in multilevel vertebroplasty.

We first try to manage this case in a medical way with rest and antibiotics, but the patient did not improve, needing surgery to remove the contaminated bonecement complex; the same as is usually done for treating infected cemented prosthesis.

Although we have enough experience in the anterior approach for spine tumors or interbody fusions, this vertebrectomy was extremely difficult because of the harder of the consistence of the bone-cement complex. We finally performed a piecemeal corpectomy, and we had to manage with severe intraoperative complications.

As stated before, we fix adjacent vertebrae with sub-laminar wiring in order to avoid contact of the implants with the vertebral bodies adjacent to L3, which seemed to have edema of possible pyogenic origin.

Postvertebroplasty infection seems to be predisposed by external factors, such as failure of the aseptic technique and inappropriate use of antibiotic prophylaxis, or by internal factors, such as immunosuppression by steroid or chemotherapy, concomitant systemic infections. It also could be by a late hematogenous unpredictable seeding, the same that might happen in hip prosthesis.

Although the rate of infection is very low compared with the enormous amount of vertebroplasty performed each year worldwide, we must take great care when performing this procedure and we have to be prepared to face complex surgery.

Although cement mixed with antibiotic is a common technique in orthopedic surgery, its use in vertebroplasty, like a prophylaxis, is not widely accepted. Kallmes and Jensen recommend its routine use and intravenous antibiotics prophylaxis only for immunosuppressed patients. ${ }^{17}$ We use routine antibiotic prophylaxis for each vertebroplasty; and in cases of oncologic patients, we try to avoid the chemotherapyinduced medullar aplasia phase before performing vertebroplasty.

\section{CONCLUSION}

Extreme asepsis of the operating theater and the technique is mandatory to prevent infection. Screening and treatment for systemic infection before vertebroplasty are strongly recommended. Use of antibiotic prophylaxis for every vertebroplasty is recommendable. In case of patients under chemotherapy, performing vertebroplasty, avoiding medullar aplasia, should be advantageous. When facing an infected vertebroplasty, initial conservative treatment with needle biopsy culture and antibiotic administration is a rational option to start. If this treatment fails, we think that surgical debridement is indicated in order to remove the infected tissue and the acrylic cement and to stabilize the spine. Although this can be an effective treatment, it could be a difficult and hazardous surgical procedure. 


\section{KEY POINTS}

- Spondylitis after vertebroplasty is a possible complication of a common procedure.

- Care must be taken to avoid contamination during vertebroplasty.

- Screening of systemic infection and prophylactic antibiotics could be considered.

- Bacteria isolation must always be tried.

- Surgical debridement and spinal stabilization could be considered as a good option if conservative treatment fails.

\section{REFERENCES}

1. Chiras J, Sola-Martinez MT, Weill A, et al. Percutaneous vertebroplasty. Rev Med Interne 1995;16:854-9.

2. Cotton A, Dewatre F, Cortet B, et al. Percutaneous vertebroplasty for osteolytic metastases and myeloma: effects of the percentage of lesion filling and leakage of methyl methacrylate at clinical follow-up. Radiology 1996;200: 525-30.

3. Harrington KD. Major neurological complications following percutaneous vertebroplasty with polymethylmethacrylate: a case report. J Bone Joint Surg Am 2001;83:1070-3.

4. Kallmes DF, Schweickert PA, Marx WF, et al. Vertebroplasty in the mid- and upper thoracic spine. AJNR Am J Neuroradiol 2002;23:1117-20.

5. O'Brien JP, Sims JT, Evans AJ. Vertebroplasty in patients with severe vertebral compression fractures: a technical report. AJNR Am J Neuroradiol 2000;21:1555-8.

6. Tohmeh AG, Mathis JM, Fenton DC, et al. Biomechanical efficacy of unipedicular versus bipedicular vertebroplasty for the management of osteoporotic compression fractures. Spine 1999;24:1772-6.

7. Jensen ME, Evans AJ, Mathis JM, et al. Percutaneous polymethylmethacrylate vertebroplasty in the treatment of osteoporotic vertebral body compression fractures: technical aspects. AJNR Am J Neuroradiol 1997;18:1897- 904.

8. Barr JD, Barr MS, Lemley TJ, et al. Percutaneous vertebroplasty for pain relief and spinal stabilization. Spine 2000;25:923-8.

9. Weill A, Chiras J, Simon JM, et al. Spinal metastases: indication for and results of percutaneous injection of acrylic surgical cement. Radiology 1996; 99:21447.

10. Chiras J, Depriester C, Weill A, et al. Percutaneous vertebral surgery: technics and indications. J Neuroradiol 1997;24:45-59.

11. Galibert P, Deramond $\mathrm{H}$. Preliminary note on the treatment of vertebral angioma by percutaneous acrylic vertebroplasty. Neurochirurgie 1987;33: 166-8.

12. Gaughen JR, Jensen ME, Scweickert PA, et al. Relevance of antecedent of venography in percutaneous vertebroplasty for the treatment of osteoporotic compression fractures. AJNR Am J Neuroradiol 2002;23:594-600.

13. Kaufmann TJ, Wald JT, Kallmes DF. A technique to circumvent subcutaneous cement tracts during percutaneous vertebroplasty. AJNR Am J Neuroradiol 2004;25:1595-6. 
14. Nussbaum D, Gailloud P, Murphy K. A review of complications associated with vertebroplasty and kyphoplasty as reported to the Food and Drug Administration medical device related web site. J Vasc Interv Radiol 2004; 15:1185-92.

15. Deramond H, Depriester C, Galibert P, et al. Percutaneous vertebroplasty with polymethylmethacrylate: technique, indications and results. Radiol Clin North Am 1998;36:533-46.

16. Padovani B, Kasriel O, Brunner P, et al. Pulmonary embolism caused by acrylic cement: a rare complication of percutaneous vertebroplasty. AJNR Am J Neuroradiol 1999;20:375-7.

17. Kallmes D, Jensen M. Percutaneous vertebroplasty. Radiology 2003;229: 27-36.

18. Yu SW, Chen WJ, Lin WC, et al. Serious pyogenic spondylitis following vertebroplasty: a case report. Spine 2004;29:E209-E211.

19. Walker DH, Mummaneni P, Roger GE Jr. Infected vertebroplasty: report of two cases and review of the literature. Neurosurg Focus 2004;17:E6.

20. Schmid KE, Boszczyk BM, Bierschneider $M$, et al. Spondylitis following vertebroplasty: a case report. Eur Spine J 2005;14:895-9.

21. Svensson O, Parment PA, Blomgren G. Orthopaedic infections by Serratia marcescens: a report of seven cases. Scand J Infect Dis 1987;19:69-75.

The manuscript submitted does not contain information about medical device(s)/drug(s).

No funds were received in support of this work. No benefits in any form have been or will be received from a commercial party related directly or indirectly to the subject of this manuscript. 


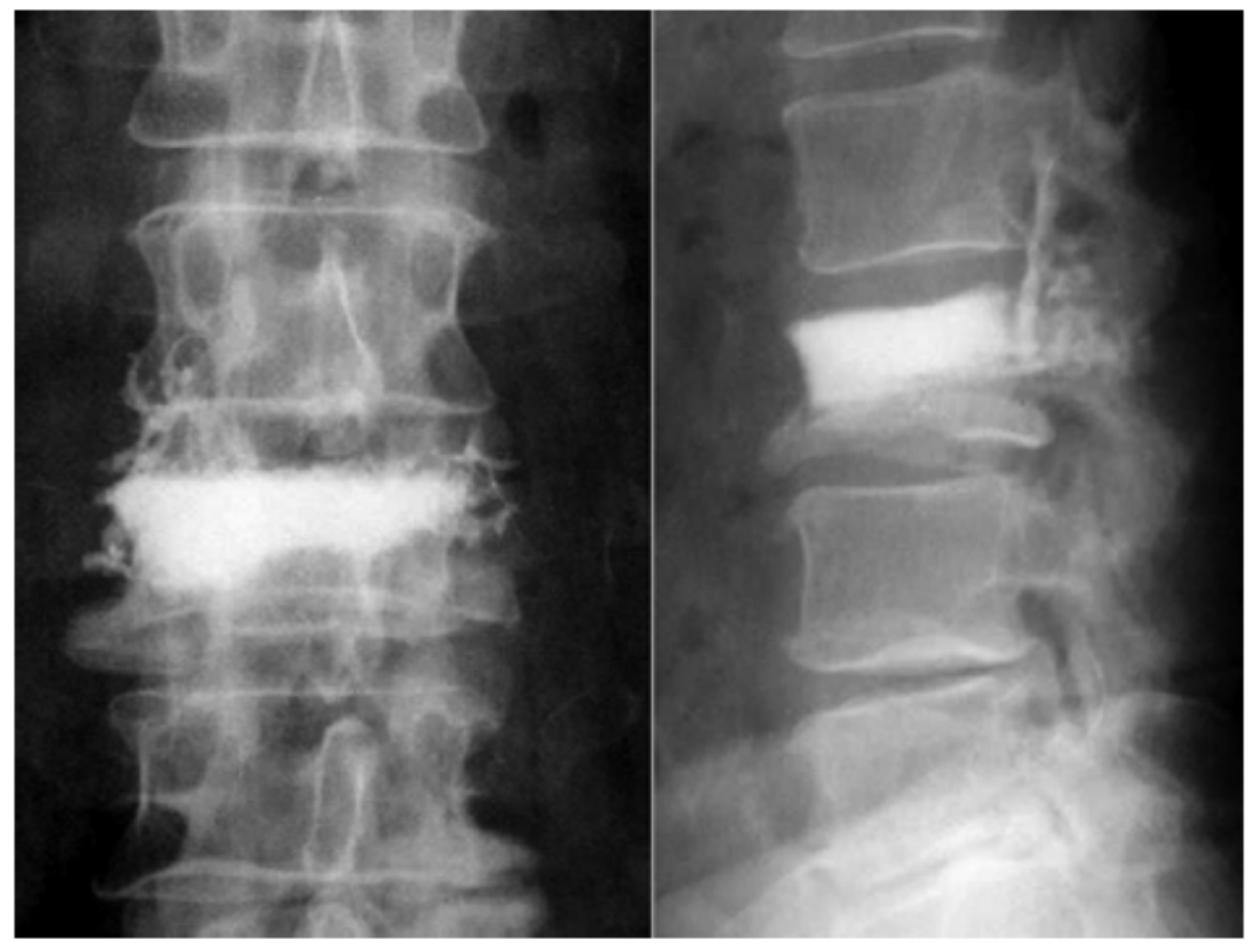

Figure 1. X-ray one month after vertebroplasty.

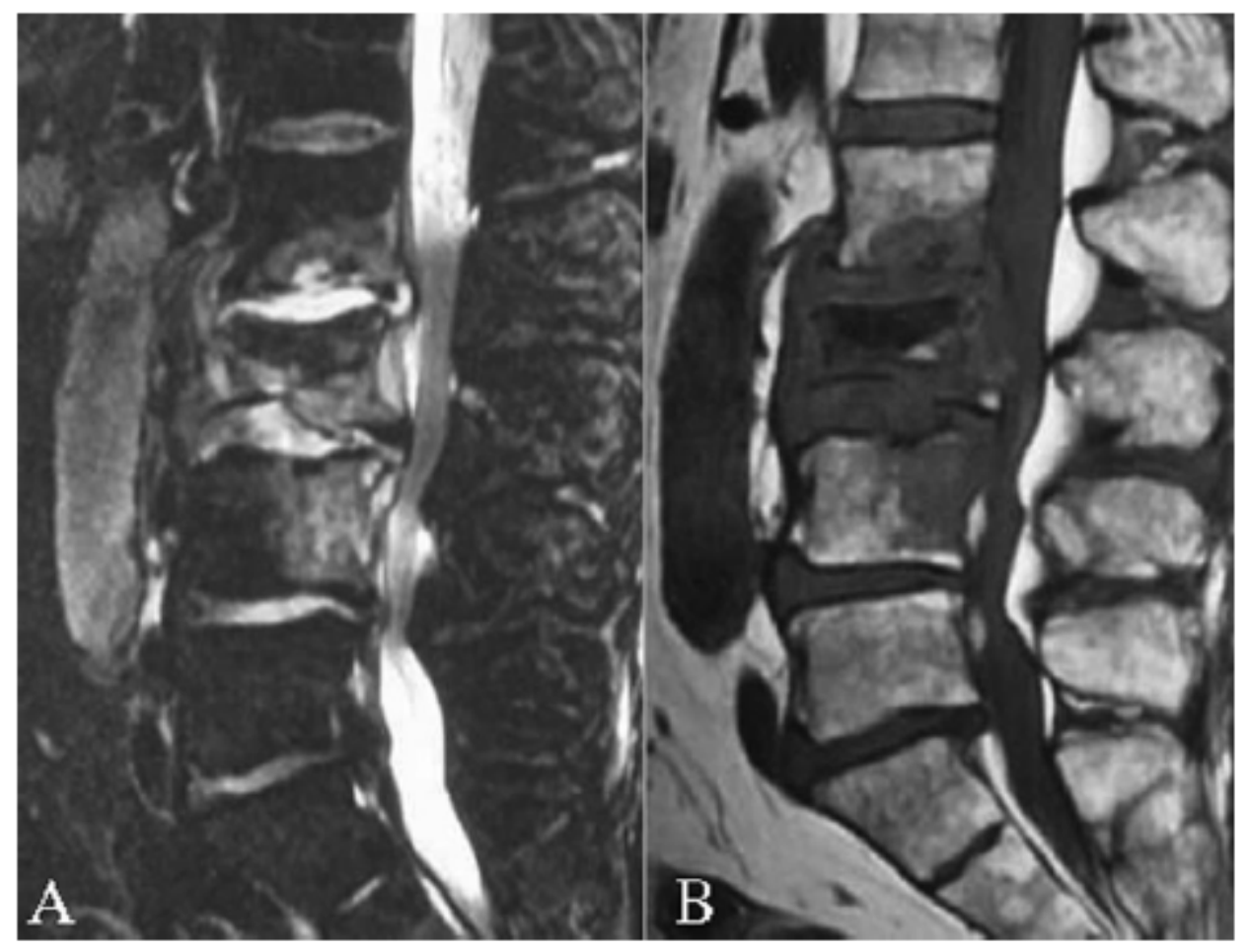

Figure 2. MRI study: (A) T2-weighted image; (B) T1-weighted image. See signs of edema in L3 and adjacent vertebrae. 


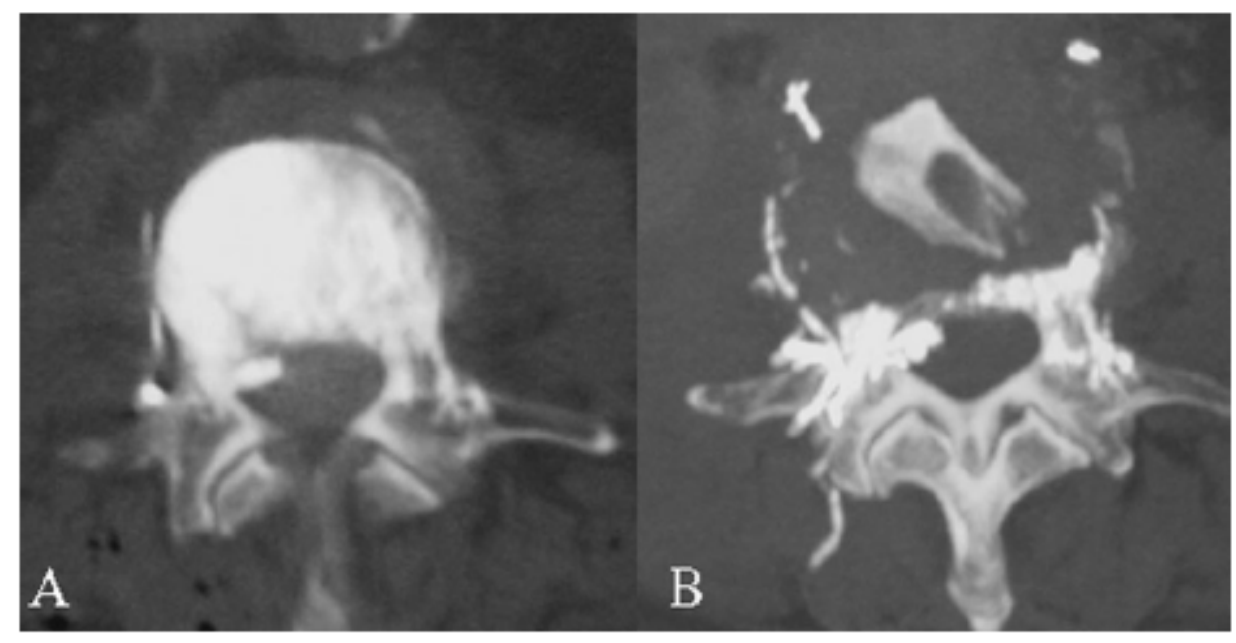

Figure 3. CT-scan: (A) L3 before corpectomy, filled with PMMA; (B) L3 after corpectomy, with the iliac graft placed like an anterior stabilizer.

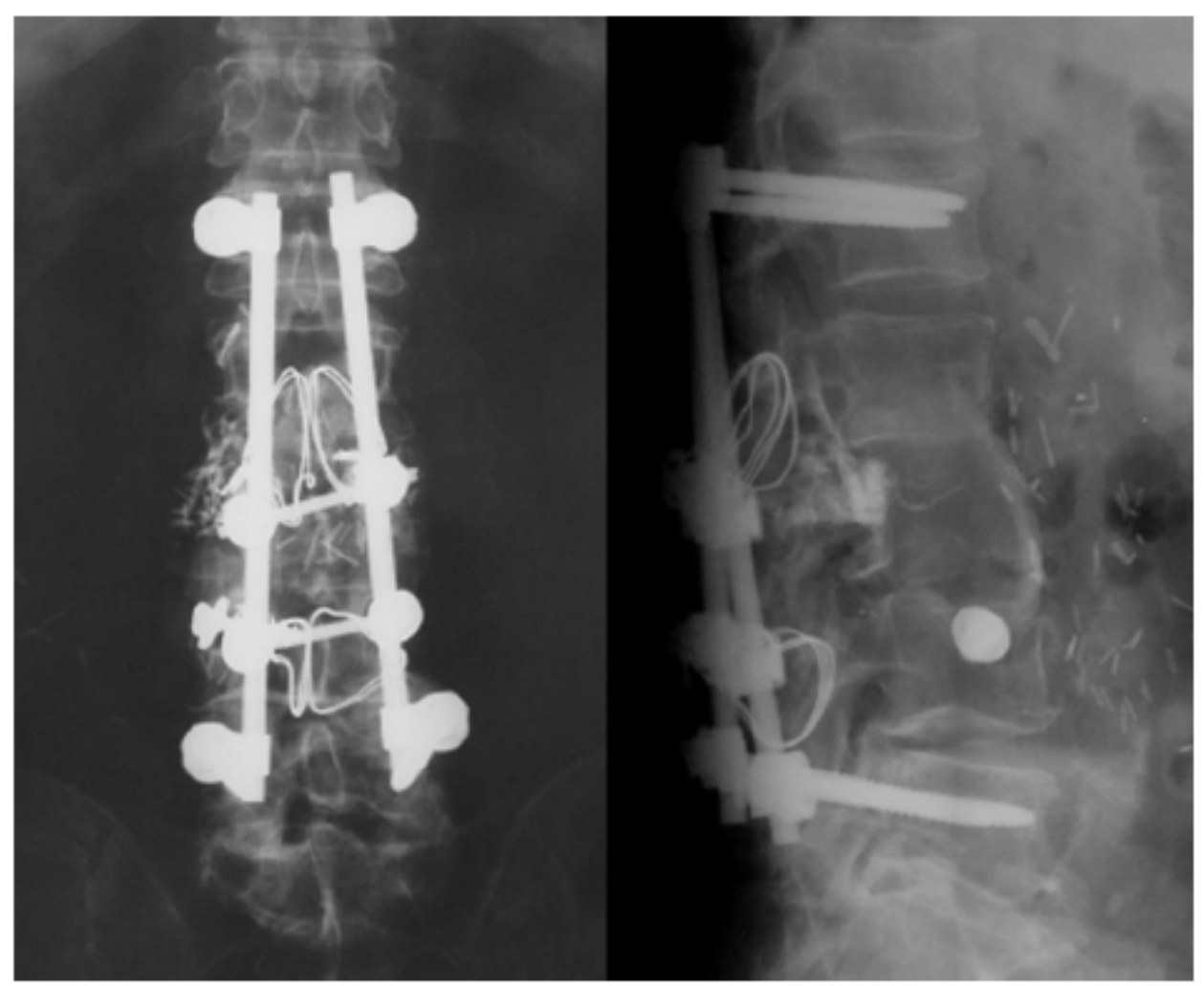

Figure 4. X-rays, 2 years of follow-up: the patient was free of pain, without signs of infection. A correct vertebral fusion was observed. See also the screw used to fix the anterior graft. 